\title{
INDEX
}

Note: legislation is indexed under the king in whose name it was promulgated. Wulfstan's writings are listed under his name, with homilies designated by numbers assigned to them by Napier or Bethurum.

Aachen, Council of 105, 219

Abbo (abbot of Fleury) 22

Abbo (monk of Saint-Germain-desPrés) 30, 208

Adalbero (bishop of Laon) 45

Admonitio generalis 91, 110

Adso (abbot of Montier-en-Der) 30, 40, 153, 208

Ælfgifu (q. of England ) 8

Flfric (abbot of Eynsham) 8, 9, 10, $16,25,26,28,29,43,63$, 95, 102, 208, 213, 214, 217 , 218,219

Book on the Old and New Testament 28, 106

Of the Eight Faults and the Truelve Abuses (De viii vitiis et xii abusivis) 113

Pastoral Letters 28-9, 73, 86, 88, 89, 90, 91, 92, 93, 94, 95, 96, 97, 98, 100, 106, 110 , $114,117,120-1,148,149$, $181,182,199,200,201$, 202, 208, 213, 215, 220

Sermon on Holy Purity (Be pare halgan clanysse) 120

Sermon on Maccabees 106

Sermon on Sexagisma Sunday (Dominica in sexagisma) 116

Sermon on the Greater Litany (Letania maiore) 104, 114, 190, 218

Sermon on the Lord's Prayer (De Dominica oratione) 105, 163, 218
Sermon on the Nativity of the Lord (Nativitatis Domini) 109

Elfric Puttoc (archbishop of York) 9, 44, 162, 197

Elfsige (bishop of Chester-le-Street) 88

Elfthryth (q. of England) 111-12

Æthelbald (abbot of WearmouthJarrow) 113

Æthelberht (k. of Kent) 4

laws of 4, 78, 223

Ethelred (k. of England) 2, 6, 11, 14, 26, 35, 38, 104, 193

laws of $5,48,88$

I Atr. 186, 195, 222, 224

III Atr. 77, 206, 224

V Atr. 14, 19, 36, 56, 57, 58, 59, 62, 69, 77, 87, 89, 91, $114,118,120,122,123$, $128,135,148,150,151$, $152,156,160,163,166$, $167,186,195,196,200$, 203, 204, 206, 210, 215

VI Atr. 14, 19, 36, 56, 57, 58, 59, 60, 61, 62, 63, 69, 73, $77,79,81,88,89,90,91$, 105, 109, 120, 122, 123, 128, 129, 135, 137, 145, $148,150,151,152,156$, $157,158,160,161,163$, $166,167,183,186,195$, 196, 199, 200, 202, 203, 204, 205, 206

VII Atr. 14, 19, 32-3, 39, 42, $51,58,130-2,184-91$ 
VIIa Atr. 14, 19, 23, 32-3, 36, 130-2, 184-91, 215

VIII Atr. 14, 19, 20, 36, 56, $58,60,70,72,75,76,77$, $78,81,82,83,84,89,97$, 99, 100, 103, 105, 123, 128, $129,135,145,149,151$, $152,156,160,161,163$, 167, 179, 183, 188, 196, 198, 200, 203, 204, 206, 210, 216

IX Atr. 14, 19, 56, 89, 123, $127,135,145,147,152$, 203, 206

X Atr. 14, 19, 56, 89, 123, 127, 128, 135, 152, 203, 206

Ethelred (k. of Northumbria) 4 Æthelstan (k. of England) 5, 6, 7, 8, 136

laws of 61

I As. 20, 136, 182, 210, 215

II As. 59, 129, 222, 223

IV As. 77, 195, 223

VI As. 59, 79, 90, 151, 158, 166, 196, 204, 224

Ethelwold (bishop of Winchester) 6, 9, 10, 27

Alcuin 4, 11, 26, 29, 30, 106, 113, 208, 220

Aldhelm (bishop of Sherborne) 4, 26

Alfred (k. of Wessex) 5, 6, 35, 39, 43, 50,56

laws of 5, 35, 56, 77, 78, 209, 222, 223

Treaty of Alfred and Guthrum 55, $56,70,222$

Alphege (archbishop of Canterbury) 7, 9, 193

Amalarius (bishop of Metz) 30, 37, 120

Regula canonicorum 86, 87, 212 Anglo-Saxon Chronicle 4, 9, 20, 193, 194, 195

Anna (Biblical prophet) 121

Ansegisus (abbot of Fontanelle) 84, 93, 94
Anti-Monastic Reaction 7, 38, 111

Asser (monk of St. David's) 26

Atto (bishop of Vercelli) 29, 123

Augustine (bishop of Hippo) 5, 181

Bath, Council of 32, 184, 189

Bede 4, 6, 26, 39

Benedict VIII (pope) 129, 193

Benedictine Office 20, 212, 216

Benedictine Rule 6, 10, 113, 120, 219, 220

Beowulf 181

Bible

Exodus 128, 167

Leviticus 173, 174-5

Numbers 109

Deuteronomy 63, 128, 163, 167, 174

Psalms 80, 109, 118, 131, 181, $187,191,206$

Proverbs 80

Isaiah 73, 107, 115, 181

Ezekiel 107, 113, 114, 115, 116

Hosea 114

Jonah 131

Matthew 63, 81, 93, 108, 109, 114, $128,135,158$

Luke 107, 108, 109, 114, 121

John 109

Acts 96, 198

I Corinthians 163

Ephesians 163, 167

I Timothy 110

Revelation 153

Boethius 106

Boniface 4, 98

Burchard (bishop of Worms) 22, 60

Brihteah 9

Byrhtferth (monk of Ramsey Abbey) 26

Byrhtnoth (earl of Essex) 16

Chalcedon, Council of 88, 149, 199

Charlemagne 4, 5

Charters 14

Chelsea, Synod of 26 
Clofesho, Council of 95

Cnut (k. of England) 2, 8, 15, 26, 35, 91, 159, 192, 193, 194, 224 laws of $5,15,44,88,143,192$

Cnut 1018 19, 143, 216

Cnut 1020 7, 15, 19, 21, 27, $34,36,57,59,60,63,85$, 90, 103, 111, 130, 146, 150, $151,158,160,166,192-6$, 204, 205, 214

\section{Cnut 102744}

I Cn. 15, 19, 36, 43, 45, 47, 49, $57,58,59,63,65,67,72$, $73,77,78,79,80,82,84$, $87,90,91,96,99,100,107$, $109,113,114,115,122$, $128,129,135,136,145$, $148,149,150,151,156$, $157,158,163,165,166$, $167,168,169,188,195$, 196, 200, 203, 204, 205, 209, 222

II Cn. 15, 19, 42, 43, 47, 49, $57,58,59,60,62,63,65$, $67,70,73,78,79,90,97$, $104,105,109,111,116$, $117,127,128,129,136$, $146,150,151,156,157$, $160,161,167,193,194$, 195, 198, 201, 203, 205, 206, 209, 222

Collectio canonum Hibernensis 19, 43, 73,105

Consilium Rispacense (Frankish Council of 798) 113

Constantine (emperor of Rome) 149

Constantinople, Council of 117, 149

Cuthbert (archbishop of Canterbury) 98

Cynesige (archbishop of Canterbury) 197

Danelaw 5, 9, 57, 59

De ecclesiasticis gradibus 93, 208, 212, 216

De medicamento animarum 108
Dunstan (archbishop of Canterbury) 6, 9, 27, 73, 99

Eadwulf (archbishop of York) 12

Edgar (k. of England) 6, 39, 71, 85, $87,111-2,136,151,163$

laws of $27,34,192,195$

II Eg. 20, 36, 58, 77, 96, 136, $151,162,163,165,166$, 195, 196, 204, 205, 209, 215,222

III Eg. 20, 62, 128, 129, 195, 209, 210, 215, 222

IV Eg. 136, 193

Edmund (k. of England) 71, 136 laws of 27,34

I Em. 136, 205, 210, 216, 222, 223

II Em. 20, 222, 224

Edmund 'Ironside' (k. of England) 8 Edward 'the Confessor' (k. of England) 8

Edward 'the Elder' (k. of Wessex) 5, 56, 195, 222, 223

Edward 'the Martyr' (k. of England) 6-7, 111

Elfwige 10

Elias (Biblical prophet) 152-3

Enham, Council of 14, 127

Enoch (Biblical prophet) 152-3

Emma (q. of England) 8

Ephesus, Council of 117, 149

'Fonthill Letter' 124

Gerard (bishop of Cambrai) 45

Gerefa 20, 44, 223

Ghaerbald (bishop of Liège) 30, 96, 97

Gregory I (pope) 5, 26, 113, 123, 151,207

Guthrum 56

Hadrian (abbot of St. Augustine's, Canterbury) 26

Haito (bishop of Basel) 182 
Handbook for a Confessor 20, 42

Harald (k. of Denmark) 194

Helen (saint) 149

Hertford, Synod of 26, 95

Hincmar (archbishop of Reims) 29, 30

Ine, laws of $59,68,71,78,89,169$, 199, 204, 209, 222, 223

Instituta Cnuti 45, 72, 76, 224

Isidore (archbishop of Seville) 163, 208

Jerome (saint) 73, 173

Jesse (bishop of Amiens) 30

John (monk of Worcester) 10

John of Salisbury (bishop of Chartres) 16

Lambarde, William 47, 65

Lantfred of Fleury (monk of Winchester) 26

Laodicea, Council of 92

'Laws of Edward the Confessor' 45, 195

Leges Henrici Primi 46, 103

Leges inter Brettos et Scottos 70

Leo IV (pope) 208

Homilia 86, 92, 93, 94

Leofsige (bishop of Worcester) 15

Lex Baiuvariorum 59

Liber Eliensis 9

Liber Wigorniensis 13

Lyfing (archbishop of Canterbury) 192

\section{Magna Carta 35}

Mainz, Synod of 60

Malcolm II (k. of Scotland) 70

Maldon, Battle of 16

Manuscripts 20-5, 207-24

Brussels, Bibliothèque Royale, 8558-63 22, 85, 89

C.C.C.C. $19021,22,65,66,72$, 73, 74, 207-9, 212, 216, 222,224
C.C.C.C. 201 23, 28, 65, 66, 68, 69, 72, 73, 85, 87, 89, 90, 94, 95, 97, 98, 100, 101, 102, 117-18, 120, 122, 130, $133,135,149,165,166$, 168, 169, 170, 173, 177, $178,184,185,195,197$, 198, 208, 214-17

C.C.C.C. 26522

C.C.C.C. $38324-5,45,55,56$, 207, 222-3, 224

C.C.C.C. 419 23, 133, 165, 168, 169,217

C.C.C.C. 421 23, 143, 218

C.U.L. Additional 3206 21, 22, 85, 101, 207

'Commonplace Books' 22-3, 24, 26, 30, 33, 49, 151

Copenhagen, Kongelige Bibliotek, Gl. Kgl. S. 1595 19, 22

London, B.L., Additional 38651 19

London, B.L., Cotton Nero A.i 21 , 34, 76, 82, 101, 102, 107, 108, 109, 116, 119, 173, $177,178,179,180,209-11$

London, B.L., Cotton Nero D.iv (Lindisfarne Gospels) 95

London, B.L., Cotton Otho B.x 133

London, B.L., Cotton Tiberius A.iii 23, 101, 127, 133, 138, 141, 165, 166, 168, 219-20

London, B.L., Cotton Vespasian A.xiv 22

Oxford, Bodleian Library, Barlow 3722

Oxford, Bodleian Library, Bodley 71822

Oxford, Bodleian Library, Hatton 113 24, 130, 133, 135, 169, 177, 178, 179, 184, 220-2

Oxford, Bodleian Library, Junius $12121,22,61,85,87,88$, 93, 94, 97, 98, 101, 102, 103, 109, 116, 117, 119, 
$121,156,173,208,211-13$, 216

Paris, Bibliothèque Nationale, lat. 3182 22, 93

Rouen, Bibliothèque Municipale, 1382 (U. 109) 22

Strood, Medway Archive and Local Studies Centre, DRc/R1 (Textus Roffensis) 25, 45, 46, $55,65,66,71,72,74,207$, 222, 223-4

York, Minster Library, Additional 1 15, 21, 34, 154, 159, 160, $162,192,214$

Monastic Reform 6, 7, 10, 11, 26-7, 30-1, 34-5, 102, 111-12

Moses 5, 174, 175, 176

Nantes, Council of 120

Nicea, Council of 117, 149

Nicene Creed 170

Norman Conquest 8, 35, 195

Northumbrian Priests' Law 20, 23, $44,56,57,84,86,88,89$, 90, 91, 92, 93, 94, 95, 97, $110,123,135,151,152$, $158,166,169,195,196$, 197-206, 215

Nowell, Laurence 47, 48, 65

Oda (archbishop of Canterbury) 26

Old English Martyrology 26

Old English Penitential (Penitential of Pseudo-Ecgbert) 86, 90, 98, 112-13, 115, 117, 198, 205, 209, 213

On the VII Ecclesiastical Ranks (De vii ordinibus ecclesiae) 73

Oscytel (archbishop of York) 12

Oswald (archbishop of York) 6, 12, 27

Parker, Matthew (archbishop of Canterbury) 47

Pax 77

Penitential of Ecgbert 114
Penitential of Halitgar 90, 209

Penitential of Pseudo-Theodore 86, 90, 91, 93, 94, 207, 216

Penitential of Theodore 93, 209

Promissio Regis 20

Quadripartitus 32, 45, 46, 61, 72, 184, 186,189

Rectitudines Singularum Personarum 20, 44, 223

Regino (abbot of Prüm) 22, 60

Regula Canonicorum 20, 49

Regularis Concordia 10, 27, 120,214, 219

Rule of Chrodegang 92

St. Brice's Day massacre 7

Sedulius Scottus 30, 43, 103, 104, 105

Stigand (archbishop of Canterbury) 12

'Sunday Letters' 44, 133, 165, 166, 219

Swerian 72, 87, 169, 179, 222, 223

Sweyn Forkbeard (k. of Denmark) 7, 8,193

Theodore of Tarsus (archbishop of Canterbury) 26

Theodosian Code 59

Theodulf (bishop of Orléans) 29, 30,37

Capitula 86, 87, 88, 89, 90, 91, 92, $93,94,95,97,110,112$, $121,157,198,202,204,217$

Thurkil the Tall 7, 130, 184, 193, 194

Trebur, Council of 93

Voluspá 60

Wanley, Humphrey 47

Wergild 71, 72, 222, 223

Whitby, Synod of 95

Wihtred, laws of 59, 78, 187, 204, 223 
William (monk of Malmesbury) 12, 46

Worcester 2, 9, 10, 12, 13, 15, 17, 21, $23,24,26,27,30,61,66$, 88, 101, 207, 209, 211,220,

Wulfmær 10

Wulfric 10

Wulfsige (bishop of Sherborne) 28, $73,89,91,92,93,94,95$, 96, 97, 98, 100, 110, 117 , 120, 148, 149, 181, 199, 200, 202, 208

Wulfstan (archbishop of York), works of

Admonition to Bishops 20, 21, 37, 91, 97, 99, 102, 107, 109, $110,112,115,119,180-3$, 210

Benedictine Office 20

Canon Law Collection 20, 73, 82, 84, 88, 89, 90, 91, 92, 93, 94, 95, 96, 97, 98, 108, 110, 117, 122, 160, 201, 202, 206, 207, 208, 211

Canons of Edgar 13, 16, 19, 21, 22, $23,24,27,28,29,37,40$, $42,48,49,55,56,57,58$, 59, 72, 82, 84, 85-100, 102, $110,114,116,117,119$, $121,122,123,135,136$, $149,151,152,156,157$, $158,162,163,165,166$, $167,168,169,180,181$, 183, 196, 197, 198, 199, 200, 201, 202, 203, 204, 205, 206, 207, 212, 216

'Compilation on Status' 13, 20, 23, $25,39,40,47,61,65-75$, 76, 101

On Ranks 20, 40, 56, 60, 65, 66, 67, 68-9, 71, 72, 75, 77, 97, 216, 224

On Wergild (Law of the Northpeople) 20, 65, 69, 70-1, 72, 216, 224

Laws of the Mercians 20, 21, 65,
70, 71-2, 209, 216, 223

Mercian Oath 20, 21, 65, 66, 70, 71, 72, 209, 216, 223

Clerical Compensation 20, 21 , $40,56,65,66,70,72-5,81$, $87,99,114,122,128,149$, 156, 179, 201, 204, 209, 216, 223

Episcopus 13, 19, 21, 37, 38, 61-4, $105,108,109,116,146$, $151,178,193,195,211$

Homilies

Bethurum 1a 11, 19, 215, 221

Bethurum 1b 11, 19, 40, 152, 213, 215, 221

Bethurum $211,19,215,221$

Bethurum 3 11, 19, 136, 143, 145, 152, 161, 215, 221

Bethurum 4 11, 19, 152, 153, 215, 221

Bethurum 5 11, 19, 79, 109, $131,147,153,156,157$, $161,175,221$

Bethurum 6 19, 116, 153, 214, 217,220

Bethurum 7 19, 81, 104, 128, $135,136,146,153,158$, 161, 170, 214, 217, 220

Bethurum 7a 19, 170, 215, 220

Bethurum 8a 19, 208, 216

Bethurum 8b 19, 81, 128, 135, 158, 163

Bethurum 8c 19, 81, 90, 91, 128, 129, 135, 137, 157, 158, 163, 216, 217, 221

Bethurum 9 19, 179, 215, 221

Bethurum 10a 19, 88, 89, 98, 104, 110, 117, 129, 136, $146,151,161,167$

Bethurum 10b 19, 81, 122, 123, 215, 221

Bethurum 1Oc 19, 62, 81, 87, 90, $96,109,122,123,135,151$, $157,158,163,168,169$, 182, 210, 215, 217, 221

Bethurum 11 19, 63, 105, 111, 
$112,116,146,195,215,221$

Bethurum 12 19, 221

Bethurum 13 14, 19, 24, 56, 63, 81, 89, 96, 104, 105, 116, $128,133-7,146,147,158$, $161,166,169,195,214$, 217, 219, 221

Bethurum 1411, 19, 221

Bethurum 15 11, 19

Bethurum 16a 19, 180

Bethurum 16b 19, 63, 107, 109, $114,180,181,215$

Bethurum 17 19, 79, 80, 107, 109, 180, 181

Bethurum 18 19, 81, 128, 135, 158,163

Bethurum 19 11, 19, 21, 23, 73, $131,147,210$

Bethurum 20 8, 14, 19, 35, 36, 47, 57, 77, 79, 80, 104, 107, 109, 122, 124, 131, 136, $137,146,147,152,156$, $161,175,177,178,180$, $181,210,215,217,221$

Bethurum 21 17, 19, 21, 23, 24, 56, 62, 75, 81, 105, 116, $123,146,147,177-9,195$, 210, 215, 216

Napier 1 19, 214, 220

Napier 19 24, 133, 165, 168

Napier 20 24, 133, 165, 168

Napier 21 24, 133, 165, 168

Napier 22 23-4, 56, 81, 89, 96, 104, 123, 128, 122, 133-7, $146,152,158,161,163$, $165,166,168,196,203$, 206, 214, 217, 219, 221

Napier 23 19, 23, 24, 45, 59, 90, 91, 92, 96, 133, 136, $151,158,162,163,164$, 165-7, 168, 196, 204, 215, 217,220

Napier 24 19, 23, 24, 37, 87, 133, 165, 168-70, 199, 215, 217

Napier 25 19, 105, 133, 165,
$168,169,215,220$

Napier 26 169, 215

Napier 27 19, 57, 147, 161, 215, 220

Napier 28213

Napier 29221

Napier 30 24, 73, 81, 122, 123, $128,133,135,158,170,221$

Napier 31221

Napier 32221

Napier 35 19, 23, 24, 33, 41, 42, 51, 110, 130-2, 147, 149, 156, 175, 184-91, 215, 221

Napier 36 19, 130, 131, 156, 184,220

Napier 37 73, 79, 80, 81, 87, 109, 114, 122, 128, 135, $156,157,158,221$

Napier 38 19, 215

Napier 40 152, 215, 217

Napier 42 104, 136, 146, 161 , 217

Napier 43 58, 59, 90, 91, 151, 158, 166, 167, 196, 204, 217

Napier 45217

Napier 46 212, 217

Napier 47 131, 143, 147, 175, 218

Napier 49 143, 218

Napier 50 19, 23, 27, 36, 40, $41,56,57,58,59,60,62$, 63, 69, 72, 77, 89, 90, 96, 99, 103, 104, 105, 111, 116, 123, 128, 129, 131, 135, 136, 143-53, 157, 158, 161, $163,164,166,167,175$, 179, 193, 195, 196, 203, 204, 206, 218

Napier 51 19, 23, 37, 57, 77, 81, 122, 127-9, 135, 136, $145,146,158,161,220$

Napier 52 19, 23, 112, 138-40, $141,154,220$

Napier 53 19, 23, 112, 122, $138,141-2,154,220$ 
Napier 57 104, 136, 146, 161, 179

Napier 58 64, 121

Napier 59 19, 21, 34, 36, 59, 73, 79, 81, 87, 90, 91, 109, $114,122,128,135,150$, $151,154-8,159,162,166$, 192, 196, 204, 214

Napier 60 18, 19, 21, 34, 57, 60, 104, 129, 136, 146, 150, $154,155,159-61,162,163$, $192,195,214$

Napier 61 19, 21, 27, 34, 36, $58,92,96,136,151,154$, $155,159,162-4,165,166$, 167, 192, 194, 195, 204, 214

Napier 62214,220

Injunction on the Behaviour of Bishops 88, 198, 199, 201-2

Institutes of Polity 3, 7, 14, 16, 17, 19, 20, 21, 23, 24, 27, 29, $30,35,37,38,41,43-4,45$, $48,49,51,56,57,60,61$, $62,63,65,67,73,77,79$, $80,81,85,87,88,89,90$, $91,94,98,101-24,127$, 129, 131, 135, 136, 138-40, $141-2,145,146,147,150$, $151,152,154,156,157$, $161,178,180,181,182$, 183, 195, 202, 203, 206,
207, 210, 211,212, 213, 215,216

Latin Homilies 19

Laws of Edward and Guthrum 13, $19,25,27,36,37,40,47$, 48, 49, 55-60, 61, 67, 70, $75,77,81,85,88,89,90$, $91,95,96,105,122,123$, $128,129,135,145,150$, $151,152,158,160,161$, $163,164,166,179,187$, $188,195,196,197,199$, 200, 203, 204, 205, 206, 222, 223

Northumbrian Church-Sanctuary 20, $21,51,79,82-4,94,95$, 200, 202, 210

On Sanctuary 20, 21, 38, 40, 49, 56, 57, 75, 76-81, 107, 109, 122, 128, 129, 135, 145, $156,157,158,179,210$

Wulfstan (Saint, bishop of Worcester) 24,88

Wulfstan Cantor 26

York 2, 4, 6, 9, 10, 11, 12, 13, 15, 16, $17,21,23,26,34,44,55$, $61,70,79,82,84,85,101$, $115,154,159,162,192$, 197, 198, 204, 209, 214 